\title{
An Integrated Decision Making Model for Evaluation of Concept Design
}

\author{
G. Green, G. Mamtani
}

The Conceptual design phase generates various design concepts and these are then evaluated in order to identify the 'Best' concept. Identifying the Best concept is important because much of the product life cycle cost is decided in this phase. Various evaluation techniques are performed so as to aid decision-making. Different criteria are weighted against concepts for the comparison. This paper describes the research being carried out at the University of Glasgow on design evaluation. It presents the Application of fuzzy logic for design evaluation and proposes an integrated decision-making model for design evaluation. This is a part of research project that aims at developing a computer tool for evaluation process to aid decision-making.

Keywords: Concept design, design evaluation, fuzzy logic.

\section{Introduction}

A typical product passes through the following life cycle (Fig. 1):

The decreased product life cycle in the present industrial scenario has led to more focus on product design. This is the result of intense competition among companies. To sustain this competition, enterprises have to pay more attention to the conceptual design phase. The conceptual design phase is one of the important phases of total design [1] and it is the phase where $70 \%$ of the product life cycle cost is decided [2].

The conceptual design phase consists of generating design concepts \& evaluation of those concepts to identify the best one out of them. The evaluation process comes into the picture for selecting the best concept. There can be a number of concepts that can be generated depending on the experience of the designers and their know-how of the component or the product. But still the number of concepts generated is limited. Hence, the best one out of these is the local optimum that is generally obtained. The 'best' is pseudo-term in the sense that one never knows how many more concepts could have been generated, which means that global optima may or may not be achieved [3]. The Local optima may or may not be the Global optima. For this to happen:

1. One has to generate a large number of concepts.

2. The evaluation process for selecting the concept has to be effective.

If the right concept is not selected, greater than necessary cost and time is used on its production. It may transpire after the product rolls out that the cost has exceeded the limits. To avoid such a situation, it is wise to take care in the conceptual design phase when selecting the concept.
The concepts are weighed and measured against criteria, and various procedures have been put forward for the final decision. One of them includes application of Fuzzy Logic as a tool to evaluate design concepts against criteria.

The next section revisits the evaluation procedure which is followed by a description of some design evaluation methods, i.e. the Method of controlled convergence and the Application of fuzzy logic [4]. A model is then proposed for computational evaluation of design concepts. This is an integrated model for decision-making and is intended to enhance the capability of novice designers.

\section{Design evaluation}

The importance of Design evaluation has been recognised over the past few years. Initially, it was applied considering a holistic approach to the product or taking a total product view [1]. Bjarnemo [3] suggested classification of general evaluation methods and proposed an integrated evaluation procedure. Green [5] used a combination of various models that leads to an integrated evaluation of concepts for the evaluation process.

Evaluation has been defined as "The activity of judging between and selecting from a range of competing design options" [5]. Design evaluation is a decision making process whereby all the concepts are enlisted and evaluated against different criteria. It plays an important role in the current scenario, as various enterprises are keen on introducing new products within a short span of time. They want to make sure that the product they introduce is best in terms of every criterion, e.g., reliability, manufacturability and cost. This fact has led to paying more attention to the evaluation process. Table 1 shows a matrix generally used for evaluation purposes.

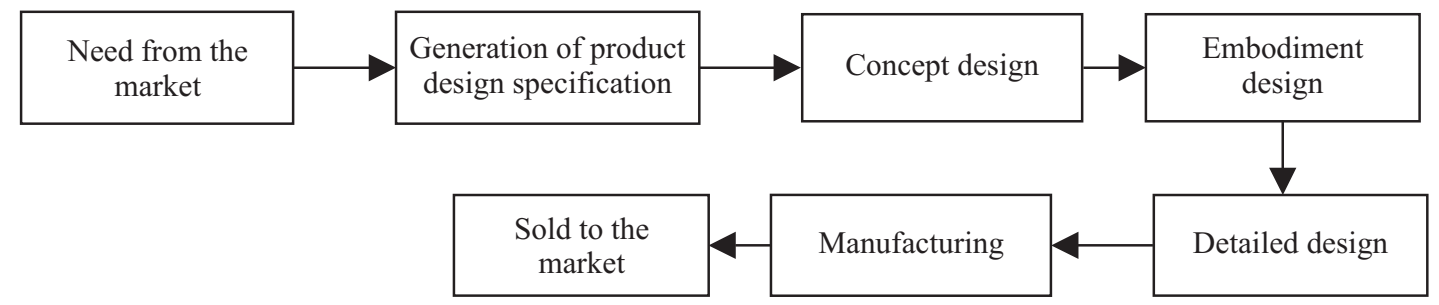

Fig. 1: Product life cycle 

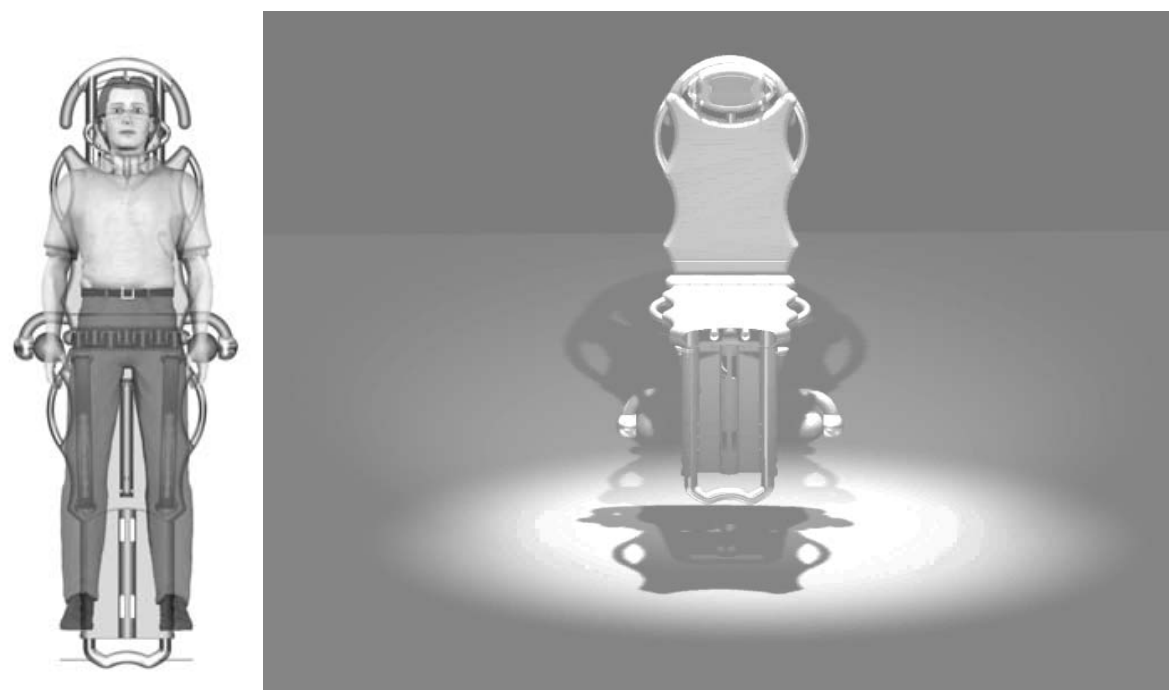

Fig. 2: Stretcher cum wheelchair

This is a matrix generated for evaluation of concepts for the design of a stretcher cum wheelchair (shown in Fig. 2). The values in the matrix are the scores provided by members of the product development team. The number of concepts generated is 3 , and there are 6 criteria.

Table 1: Concept/Criteria evaluation matrix

\begin{tabular}{|l|c|c|c|}
\hline Criteria & Concept 1 & Concept 2 & Concept 3 \\
\hline Portable & 6 & 7 & 8 \\
\hline Safe \& reliable & 6 & 6 & 7 \\
\hline Easy to use & 7 & 7 & 7 \\
\hline Flexible & 7 & 8 & 8 \\
\hline Good aesthetics & 8 & 7 & 8 \\
\hline Good ergonomics & 8 & 7 & 8 \\
\hline
\end{tabular}

\section{Method of controlled convergence}

For selecting the right concept, the method of Controlled convergence is an effective evaluation tool. Alternate convergent and divergent thinking forms the basis of this method. The reasoning is followed by a reduction in the number of concepts and then new concepts are generated. This alternate reduction and generation of concepts is followed until the final concept to be considered is arrived at.

Initially, a product design specification is generated which in turn forms the basis for generating of various concepts. These concepts are then compared using an evaluation matrix leading to a reduction in the number of concepts. After this, the concept generation process is reapplied and new concepts are added. Again a concept comparison and re-reduction process is run through, so as to filter the concepts or aid final selection. This reduction and generation of concepts is followed until the Best concept is finally selected. Fig. 3 depicts this model.

\section{Fuzzy logic and its application to design evaluation}

\subsection{Fuzzy and crisp sets}

Fuzzy sets are used when the information available is fuzzy or vague. For example, when we say that the car has a high mileage, we are not sure about the exact mileage the car has. This is a fuzzy expression. There may be several cars with a high mileage, but the degree to which they belong to this set of cars (i.e. High mileage cars) is variable. As such, each element of a fuzzy set belongs to it with a certain degree of

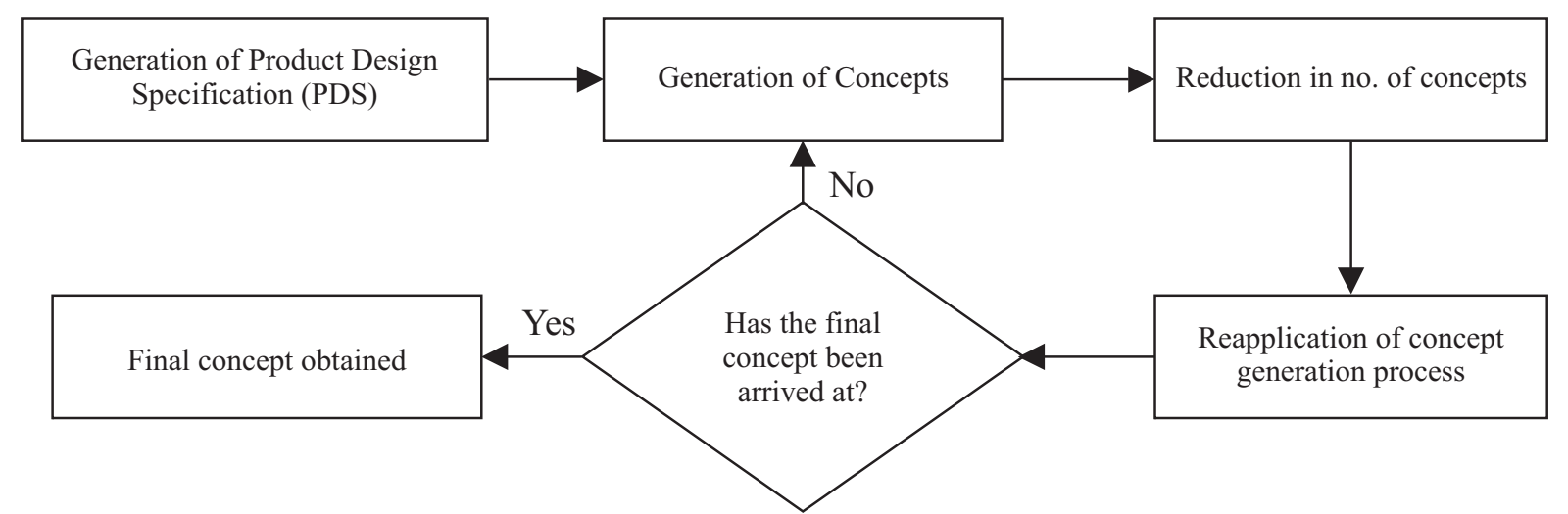

Fig. 3: Method of Controlled convergence (after pugh) 
membership. Thus, a fuzzy statement is not either true or false but may be partly true or partly false to some extent.

Crisp sets or classical sets are special cases of fuzzy sets when the degree of membership of the element is either 0 or 1. It is based on the logic that uses one of the two values: true or false. For example, when we say that all cars with a mileage of more than 10 miles/litre are considered to have a high mileage and, below this, 'not high' mileage. This means that a car can have either a high mileage or 'not high' mileage.

A crisp set $A$ of Universe $X$ is defined by the characteristic function $f_{A}(x)$

$$
\begin{aligned}
& f_{A}(x): X \rightarrow[0,1] \\
& f_{A}(x)=1, \text { if } x \in A \\
& 0, \text { if } x \notin A .
\end{aligned}
$$

A fuzzy set $A$ of universe $X$ is defined by membership function $m_{A}(x)$

$$
m_{A}(x): \mathrm{X} \rightarrow[0,1]
$$

Where,
(a) $m_{A}(x)=1$ if $x$ is totally in $A$,
(b) $m_{A}(x)=0$ if $x$ is not in $A$,
(c) $0<m_{A}(x)<1$ if $x$ is partly in $A$.

In case $\mathrm{a}$ and $\mathrm{b}$ above, a fuzzy set becomes a crisp set.

\subsection{Linguistic values and fuzzy rules}

Linguistic values are used to describe the fuzziness of the situation. For example, when we say that a person is a quick runner, the linguistic value is quick. It does not actually show how quick the person is and therefore it is fuzzy in this respect. Some other examples of linguistic values are tall, short, good, high, better, etc. These values are used to define the fuzzy rules, such as: "If the distance is more, the time taken is also more" or "If the speed is high, time taken is less".

Fuzzy rules, in turn are the expert rules that drive the conclusion. These must already to be prepared and stored in the database. These rules are generated with the help of experts, heuristics, various books, journals and databases.

\subsection{Fuzzy set operations}

Let $A$ and $B$ be two fuzzy sets and $X$ be the universe of discourse. The following are a few common operations applied on fuzzy sets:

$$
\begin{array}{ll}
\text { Complement } & m_{-A}(x)=1-m_{A}(x) \\
\text { Union } & M_{A \cup B}(x)=\max \left[m_{A}(x), m_{B}(x)\right] \\
\text { Intersection } & M_{A \cap B}(x)=\max \left[m_{A}(x), m_{B}(x)\right]
\end{array}
$$

These operations are useful when fuzzy rules are applied. There are combinations of Fuzzy rules that use the above operations for the generation of output.

\subsection{Fuzzy logic inference}

The fuzzy inference technique discussed here is the Mamdani style inference [6], which is the most commonly used inference. This inference comprises the following steps:

1. Fuzzification:

Input of Crisp values and allocating them to the fuzzy sets they belong to, thereby determining their membership value.

2. Rule application:

The fuzzy rules are then applied on the fuzzified inputs. These fuzzy rules make use of fuzzy operators (AND or OR) and arrive at the solution.

3. Output generation:

The solution arrived at after the rule applications provides the unified outputs that are combined into a single output fuzzy set.

4. Defuzzification:

This output value arrived at is finally defuzzified so as to get the final crisp result.

\subsection{Application of fuzzy logic to evaluation}

Referring to Table 1 of the evaluation matrix, fuzzy logic is applied to fill the matrix. It means in place of scores allocated to the matrix, now fuzzy linguistic values are allocated [7]. Table 2 shows the same example with fuzzy values in the matrix. This is the same example of the design of a stretcher cum wheelchair. The number of concepts generated is 3 , and there are 6 criteria.

The inference discussed in the previous section is used for decoding this matrix and for getting the final crisp output. The final defuzzified result gives a value for each of the above 3 concepts, and the one with the largest value is finally selected.

Table 2: Use of fuzzy values for weighting criteria

\begin{tabular}{|l|l|l|l|}
\hline Criteria & Concept 1 & Concept 2 & Concept 3 \\
\hline Portable & Good & Very good & Excellent \\
\hline Safe \& reliable & Good & Good & Very good \\
\hline Easy to use & Very good & Very good & Very good \\
\hline Flexible & Very good & Excellent & Excellent \\
\hline Good aesthetic & Excellent & Very good & Excellent \\
\hline Good ergonomics & Excellent & Very good & Excellent \\
\hline
\end{tabular}

\section{Proposal of an integrated decision making model for evaluation}

As seen in the previous sections, fuzzy linguistic values are allocated by human beings. These fuzzified values are used for fuzzy inference to get the solution. Such a model is as follows (Fig. 4).

Now such a model requires a lot of intervention by humans, and the result depends a lot on the experience of the designer. If the designer is experienced, the chance of getting a good solution is increased because of the allocation of appropriate linguistic values provided by him. But if the designer is a novice, chances are that he may not be able to provide appropriate fuzzy variables and that the solution is affected due to this.

Henceforth, a model is sought to take care of such cases. In this model, the score values are provided by computation. 


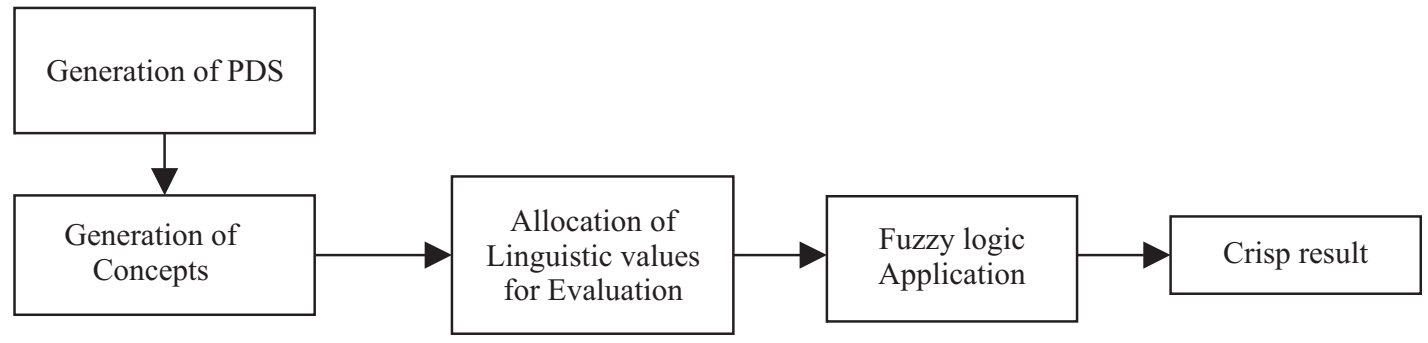

Fig. 4: Fuzzy logic application process

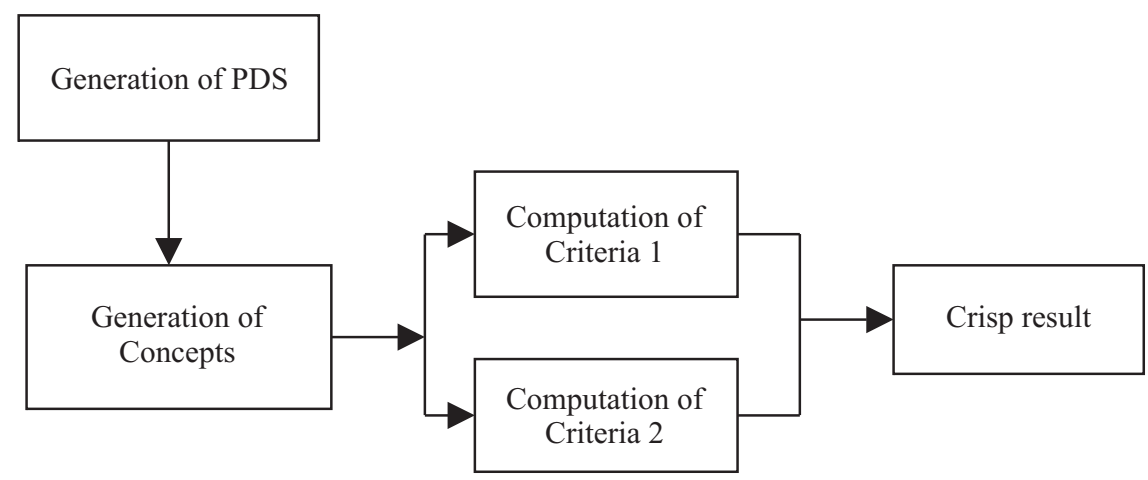

Fig. 5: Proposed computational model

This may be done by calculating the criteria in question to get the score values so as to fill their respective positions in the evaluation matrix. This computational model is depicted in Fig. 5 for a concept evaluation with 2 criteria only.

For such a model, rules have to be discovered and generated for the calculation of various criteria used in different systems. This means that if the criteria are, for example reliability, manufacturability and ease of assembly, a specific product shall be considered for which the calculations are to be done.

A questionnaire has been prepared and sent to various Scottish industries involved in design to know the practical interest of industry in such a proposed model. It contains various questions on the importance of Evaluation activity within their company and inclination towards computational evaluation. The results will be reported elsewhere in due course.

\section{Conclusion}

The previous sections have shown the importance of Evaluation procedures and the requirements of various models to enhance the capability of novice designers. It has also shown how fuzzy logic application to evaluation helps in the selection of concepts. It is particularly helpful in the Conceptual design phase, which lacks the information content. The model proposed is helpful in the sense that it will compensate for the lower experience of novice designers. It will, in due course, be subjected to experimental testing to determine its validity.

Future work involves evaluating concepts with this model and the criteria considered will be reliability, manufacturability and ease of assembly. Initially, it will be tested with a specific product, and then work will be done towards generali- sation of the model for products with more or less common attributes.

\section{References}

[1] Pugh S.: Total Design: Integrated Methods for Successful Product Engineering. Workingham, 1991.

[2] Nevins J. L. et al: Concurrent Design of Product and Processes. Daniel E. Whitney, New York, 1989.

[3] Bjarnemo R.: Towards a computer implementable evaluation procedure for the mechanical engineering design process. Lund, 1994.

[4] Zadeh L. A.: "Fuzzy Sets". Information and Control, Vol. 8, (1965), p. 338-353.

[5] Green G.: "Towards an Integrated Design Evaluation Tool (IDE) Tool”. Acta Polytechnica, Vol. 40 (2000), p. 50-56.

[6] Negnevitsky M.: Artificial Intelligence- A Guide to Intelligent Systems. Harlow, 2001.

[7] Wang J.: "A fuzzy outranking method for conceptual design evaluation". International Journal of Production Research, Vol. 35 (1997), p. 995-1010.

Dr. Graham Green

e-mail: g.green@mech.gla.ac.uk

Mr. Girish Mamtani

Mechanical Engineering Department

James Watt Building

University of Glasgow

Glasgow, G12 8QQ, Scotland, UK 